\title{
Enhanced optical characteristics of light emitting diodes by surface plasmon of Ag nanostructures
}

Lee-Woon Jang

Jin-Woo Ju

Ju-Won Jeon

Dae-Woo Jeon Jung-Hun Choi

Seung-Jae Lee Seong-Ran Jeon Jong-Hyeob Baek

E. Sari

H. V. Demir

Hyung-Do Yoon

Sung-Min Hwang

In-Hwan Lee 


\title{
Enhanced optical characteristics of light emitting diodes by surface Plasmon of Ag nanostructures
}

\author{
Lee-Woon Jang ${ }^{1}$, Jin-Woo Ju ${ }^{1}$, Ju-Won Jeon ${ }^{1}$, Dae-Woo Jeon ${ }^{1}$, Jung-Hun Choi ${ }^{1}$, Seung-Jae Lee ${ }^{2}$, \\ Seong-Ran Jeon ${ }^{2}$, Jong-Hyeob Baek ${ }^{2}$, E. Sari ${ }^{3}$, H. V. Demir ${ }^{3}$, Hyung-Do Yoon ${ }^{4}$, Sung-Min Hwang ${ }^{4}$, \\ and In-Hwan Lee ${ }^{1 *}$ \\ ${ }^{1}$ School of Advanced Materials Engineering and Research Center of Advanced Materials \\ Development, Chonbuk National University, Jeonju 561-756, Korea \\ ${ }^{2}$ LED Device Team, Korea Photonics Technology Institute, Gwangju 500-779, Korea \\ ${ }^{3}$ Nanotechnology Research Center, Department of Electrical and Electronics Engineering, Bilkent \\ University, Ankara, TR-06800 Bilkent, Turkey \\ ${ }^{4}$ Nano scale Quantum Devices Research Center, Korea Electronics Technology Institute, Gyeonggi \\ 463-816, Korea
}

\begin{abstract}
We investigated the surface plasmon coupling behavior in $\mathrm{InGaN} / \mathrm{GaN}$ multiple quantum wells at $460 \mathrm{~nm}$ by employing Ag nanostructures on the top of a roughened p-type GaN. After the growth of a blue light emitting diode structure, the p-GaN layer was roughened by inductive coupled plasma etching and the Ag nanostructures were formed on it. This structure showed a drastic enhancement in photoluminescence and electroluminescence intensity and the degree of enhancement was found to depend on the morphology of Ag nanostructures. From the time-resolved photoluminescence measurement a faster decay rate for the Ag-coated structure was observed. The calculated Purcell enhancement factor indicated that the improved luminescence intensity was attributed to the energy transfer from electron-hole pair recombination in the quantum well to electron vibrations of surface plasmon at the Ag-coated surface of the roughened $\mathrm{p}-\mathrm{GaN}$.
\end{abstract}

\footnotetext{
* Corresponding author.

E-mail address : ihlee@jbnu.ac.kr
}

Quantum Sensing and Nanophotonic Devices VIII, edited by Manijeh Razeghi, Rengarajan Sudharsanan, Gail J. Brown, Proc. of SPIE Vol. 7945, 794511 - (c) 2011 SPIE · CCC code: 0277-786X/11/\$18 · doi: 10.1117/12.869465 


\section{INTRODUCTION}

GaN-based light-emitting diodes (LEDs) with $\mathrm{InGaN} / \mathrm{GaN}$ multiple quantum well (MQW) structures, ranging from short-wavelength to long-wavelength part of the visible spectrum have been intensely developed [1-3]. However, crystal defects, dislocation and piezoelectric fields induce the non-radiative recombination energy in the InGaN/GaN MQW structures, which suppress the external quantum efficiency of GaN-based LEDs [1-4]. To achieve the high-efficiency LEDs, it is necessary to increase the optical extraction efficiency and internal quantum efficiency. Many interesting studies have been proposed to achieve this, such as the application of surface roughness, photonic crystal, carrier confinement and surface plasmon (SP) [4-10]. Especially, SP has attracted recent research interest of GaN-based LEDs requiring high quantum efficiency.

When a metal deposited on the surface of semiconductor (dielectric), the electro-magnetic field by oscillation of electron gas in metal is formed, the surface plasmon. If the SP energy matched well with the photon energy of LEDs, electron-hole pairs can transfer to the SP mode beside the radiative- and non-radiative recombination process in the active layer of LEDs [11-13]. In other words, SP provides the additional emission process by energy coupling between the SP and electron-hole pair, and suppress the non-radiative recombination process of injected carrier in the active layer. As a result, the internal quantum efficiency of LEDs can be increased. From these SP mechanisms, the joint researchers of CalTech and Nichia firstly reported the SP effect at the InGaN based blue LED structure [11]. The spacer thickness is a important factor for SP energy transfer, and it can be determined to calculate the penetration depth of SP. The penetration depth for the effective energy coupling into the semiconductor is given by $Z=\lambda / 2 \pi\left[\left(\varepsilon_{\mathrm{GaN}}^{\prime}-\varepsilon_{\text {metal }}^{\prime}\right) / \varepsilon_{\mathrm{GaN}}^{\prime}\right]^{2 / 2}$, where $\varepsilon_{\text {GaN }}^{\prime}$ and $\varepsilon_{\text {metal }}^{\prime}$ are the real part of the dielectric constants of the semiconductor and metal, respectively [11]. Using the real part of the dielectric constant of the $\mathrm{GaN}$ and $\mathrm{Ag}$ metal, the penetration depth $\mathrm{Z}$ at an emission energy of $2.7 \mathrm{eV}(459.2 \mathrm{~nm})$ for a blue LED was estimated to be $42 \mathrm{~nm}$ for SP-MQW coupling, i.e., the Ag structure should be within $42 \mathrm{~nm}$ from the active layer of LEDs. However $\mathrm{p}-\mathrm{GaN}$ can be substituted for the spacer layer in the conventional LEDs with thickness greater than $76 \mathrm{~nm}$ in a blue LEDs to obtain the efficient carrier injection [4]. But the thickness of spacer layer is found to be too thick for the energy transfer by SP-MQW coupling. Till date, there has been no report on the SP energy coupling deposited on the conventional LEDs having thick p-GaN.

In this paper, we approached a structural way to obtain the energy coupling by SP as well as the carrier injection of the conventional LEDs. We fabricated a $150 \mathrm{~nm}$-thick p-GaN layer for the carrier injection and partially etched p-GaN layer for the efficient SP coupling. This structure showed a remarkable increase in PL and EL intensities. From the time resolved PL (TRPL) measurements, a faster decay rate and the calculated Purcell enhancement factor of partially etched LED with Ag nanostructures represented the energy transfer by SP-MQW coupling. 


\section{EXPERIMENTAL PROCEDURE}

The InGaN/GaN MQW epitaxial structure was grown by metal organic chemical vapor deposition (MOCVD) technique. Trimethylgallium (TMGa), trimethylindium (TMIn) and $\mathrm{NH}_{3}$ were used as precursors for $\mathrm{Ga}$, In and $\mathrm{N}$ respectively. A thermal annealing of c-plane sapphire substrate was carried out at $1100{ }^{\circ} \mathrm{C}$ for $10 \mathrm{~min}$, followed by the growth of a low temperature GaN buffer layer. A $1 \mu \mathrm{m}$-thick undoped GaN layer and a $2 \mu \mathrm{m}$-thick n-type GaN layer were grown at $1060{ }^{\circ} \mathrm{C}$. Then, five pairs of InGaN/GaN MQW were grown on high quality GaN epitaxial layers. The $\mathrm{GaN}$ barriers and $\mathrm{InGaN}$ wells were grown at temperatures of $850{ }^{\circ} \mathrm{C}$ and $750{ }^{\circ} \mathrm{C}$ respectively. Two types of GaN layers were grown as the spacer layer between metal and MQWs, (i) $10 \mathrm{~nm}$-thick undoped GaN layer was directly grown on MQW to investigate the SP-MQW energy coupling by the Ag nanostructures and (ii) $150 \mathrm{~nm}$-thick p-GaN layer was grown on for electrical characterization. The schematic diagram of the GaN LEDs structures are shown in Fig. 1 (a) and Fig. 1 (b). In order to fabricate the partially etched p-GaN layer, a $100 \mathrm{~nm}$-thick $\mathrm{SiO}_{2}$ and $10 \mathrm{~nm}$-thick Ni mask were deposited on the sample by plasma-enhanced CVD and e-beam evaporator [14]. The sample was subsequently annealed at $800{ }^{\circ} \mathrm{C}$ for 1 min under $\mathrm{N}_{2}$ ambient to form the Ni clusters. Finally, the $\mathrm{SiO}_{2}$ and p-GaN layer were etched for $120 \mathrm{sec}$ using an ICP-reactive ion etching system. The etch depth of the p-GaN was $\sim 130 \mathrm{~nm}$. For the fabrication of $\mathrm{Ag}$ nanostructure, Ag thin film was deposited on the etched p-GaN layer of LED and subjected to rapid thermal annealing (RTA) under Ar ambient at $250^{\circ} \mathrm{C}$ for $10 \mathrm{~min}$.

The surface morphology of the deposited Ag was investigated by scanning electron microscopy (SEM, HITACHI S4300SE). PL and EL measurements were carried out to study the optical properties. The electrical luminescence characteristics were quantified through the back side photo detector by on-wafer probing of the devices. A $25 \mathrm{~mW}, \mathrm{He}-\mathrm{Cd}$ laser $(325 \mathrm{~nm})$ was used as the excitation source for the room temperature (RT) PL measurements. We carried out RTTRPL measurements (using PicoQuant PicoHarp 300) to clarify the SP-MQW coupling. The optical apparatus consists of a commercially available InGaN/GaN based $375 \mathrm{~nm}$ laser diode used in pulsed mode operation, a monochromator, a photomultiplier tube, a high speed photodetector and controller electronics. All decay lifetimes are calculated by the software of Pico-Quant (FLUOFIT).

\section{RESULTS and DISCUSSION}

Figure 2 (a)-(d) shows the SEM images of the Ag nanostructures formed on the $10 \mathrm{~nm}$ undoped GaN spacer after annealing the Ag films with thickness of $10 \mathrm{~nm}, 15 \mathrm{~nm}, 20 \mathrm{~nm}$ and $25 \mathrm{~nm}$ at $250{ }^{\circ} \mathrm{C}$ for 10 min. A variety of morphologies ranging from irregular islands to regular clusters were obtained. The patch-shaped large Ag islands were noticed for the $25 \mathrm{~nm}$-thick Ag film and the curved rod-shaped Ag clusters were observed for the 15-20 nm-thick Ag films. The $10 \mathrm{~nm}$-thick Ag film resulted in the formation of regularly shaped, densely distributed Ag nanostructures with an average size of $\sim 100 \mathrm{~nm}$ in diameter.

The front side PL responses from the MQW with the Ag nanostructures obtained from 10-25 nm-thick Ag films are shown in Fig. 3. Multiple interference peaks were clearly observed for the bare LEDs, indicating the presence of Fabry- 
Perot (FP) effect caused by the mirror-like surface. However, the PL spectra of all the Ag-coated samples showed weak FP effects due to the light scattering caused by the Ag nanostructures at the Ag-GaN interface $[15,16]$. Compared with the uncoated sample, the MQW coated with 10 and $25 \mathrm{~nm}$-thick Ag films exhibited the decrease in PL intensities, whereas the $15 \mathrm{~nm}$-thick Ag film showed a slight increase in PL intensity. In a sharp contrast, the sample coated with 20 nm-thick Ag films showed a remarkable increase in the PL intensity by about $80 \%$. In the SP mechanism, the phase matching between SP and photon energy is the important factor for the effective light emission [17]. The phase matching is controlled by the size and shape of the nanostructures as well as dielectric property of metal $[18,19]$. With the increasing Ag thickness, the nanostructure size after the thermal annealing process was increased and the SP resonance wavelength gets red-shifted. The suitable thickness of Ag layer for efficient SP coupling at $460 \mathrm{~nm}$ is a $20 \mathrm{~nm}$ and it showed a good phase matching condition [20]. The results are found to be consistent with our enhanced PL intensity.

Based on these results, the $20 \mathrm{~nm}$-thick Ag film layer was formed on the LEDs with a $150 \mathrm{~nm}$-thick p-GaN layer by depositing and annealing as shown in Fig. 1 (b). Figure 4 shows the EL spectra of different LED structures, the bare LED without Ag (not etched) (sample A), the ICP-etched LED without Ag (sample B), and the Ag-coated LED after ICP (sample C). The peak wavelengths of the EL spectra were observed at 465, 460 and $462 \mathrm{~nm}$ for sample A, sample B and sample $\mathrm{C}$ at operating current of $20 \mathrm{~mA}$. The inset of Fig. 4 shows the tilted SEM image of sample C. The forward voltages were 7.8, 7.7 and $8.3 \mathrm{~V}$ for the sample $\mathrm{A}, \mathrm{B}$ and $\mathrm{C}$ respectively, at the operating current of $20 \mathrm{~mA}$. The integrated EL intensity of the sample B compared with sample A was enhanced about $10 \%$. The enhancement of the sample $\mathrm{B}$ is due to the increase of the light extraction by partially etched p-GaN [10, 21]. However, the integrated EL intensity of the sample $\mathrm{C}$ with a higher forward voltage was enhanced about $53 \%$. The significant EL enhancement is related to the SP energy coupling by the deposited Ag nanostructures on thin $\mathrm{p}-\mathrm{GaN}$ after ICP etching as well as the increase of the light extraction by ICP etching.

The TRPL measurements were performed to investigate the SP coupling at the maximum emission wavelength of each sample and the results are shown in Fig. 5. After etching LEDs by ICP, the decay time was observed to decrease from $5.89 \mathrm{~ns}$ to $5.1 \mathrm{~ns}$ due to the increase of the non-radiative recombination rate in $\mathrm{p}$-GaN layer. The relatively larger non-radiative recombination rate was caused by the ICP etching damages [21]. The average decay curve of the sample C was shorter than the sample A and B which was 3.3 ns. However it shows a multi-stage decay behavior. The fast and slow decay time of the sample $\mathrm{C}$ were found to be $290 \mathrm{ps}$ and $4.16 \mathrm{~ns}$, respectively. Normally, the fast decay time results from exciton recombination while the slow decay time attributed to localized carrier recombination by the quantumconfined Stark effect [21]. When the exciton energy transferred to the SP, the exciton energy of the MQW (fast decay) is close to the electron vibration energy of the SP [4, 11-13]. It means that the 290 ps decay time is obtained from the SP energy coupling of $\mathrm{Ag}$ nanostructures on the partially etched $\mathrm{p}-\mathrm{GaN}$ layer. To describe the increase in spontaneous emission rate $\left(R_{\mathrm{se}}\right)$, the increase in Purcell enhancement factor $\left(F_{P}\right)$ was calculated using the equation:

$$
F_{P}(\omega)=\tau_{\mathrm{PL}}(\omega) / \tau_{\mathrm{PL}}^{*}(\omega)=k_{\mathrm{PL}}{ }^{*}(\omega) / k_{\mathrm{PL}}(\omega)
$$


where $k_{\mathrm{PL}}(\omega)$ and $k_{\mathrm{PL}}{ }^{*}(\omega)$ are the original and changed PL decay rate, respectively [22]. From the equation, the $F_{P}$ value was found to be 1.54 at emission energy of $2.68 \mathrm{eV}(462 \mathrm{~nm})$ for the EL improvement of the sample C. Therefore, the high $F_{P}$ indicates that the improved EL intensity is attributed to the increase in spontaneous emission rate suggesting the effective coupling of SP-MQW.

\section{Conclusion}

We investigated the SP coupling behavior in InGaN/GaN MQWs by employing Ag nanostructures on the top of roughened p-type GaN. From the results of PL and EL measurements, the luminescence efficiency was enhanced by $80 \%$ and $53 \%$ for an input current of $20 \mathrm{~mA}$. The calculated Purcell enhancement factor by faster decay time of partially etched LED with Ag nanostructures indicated that the enhanced luminescence intensity was attributed to the energy transfer from electron-hole pair recombination in MQW layer to electron vibrations of SP at the Ag-coated surface on the roughened $\mathrm{p}-\mathrm{GaN}$.

\section{Acknowledgement}

This work was supported by Basic Science Research Program through the National Research Foundation of Korea (NRF) funded by the Ministry of Education, Science and Technology (R01-2007-000-11177-0) 


\section{References}

[1] S. Nakamura, T. Mukai, and M. Senoh, "Candela-class high-brightness InGaN/AlGaN double-heterostructure bluelight-emitting diodes", Appl. Phys. Lett. 64, 13 (1994).

[2] S. Nakamura, "The roles of structural imperfections in InGaN-based blue light-emitting diodes and laser diodes", Science 281, 956 (1998).

[3] Z. Lei, G. Xia, L, Ting, G. Xiaoling, L. Q. Ming, and S. Guangdi, "Color rendering and luminous efficacy of trichromatic and tetrachromatic LED-based white LEDs", Microelectron. J. 38, 1 (2007).

[4] M. K. Kwon, J. Y. Kim, B. H. Kim, I. K. Park, C. Y. Cho, C. C. Byeon, and S. J. Park, "Surface-plasmon-enhanced light-emitting diodes", Adv. Mater. 20, 1253 (2008).

[5] W. Liu, A. A. Balandin, C. H. Lee, and H. Y. Lee, "Increased thermal conductivity of free-standing low-dislocationdensity GaN films", Phys. Stat. Sol. (a) 202, 12 (2005).

[6] I. H. Lee, A. Y. Polyakov, N. B. Smirnov, A. V. Govorkov, A. V. Markov, and S. J. Pearton, "Electrical and recombination properties and deep traps spectra in MOCVD ELOG GaN layers”, Phys. Stat. Sol. (c) 3, 6 (2006).

[7] J. W. Ju, H. S. Kim, L. W. Jang, J. H. Baek, D. C. Shin, and I. H. Lee, "A well protection layer as a novel pathway to increase indium composition: a route towards green emission from a blue InGaN/GaN multiple quantum well", Nanotechnol. 18, 295402 (2007).

[8] M. K. Kwon, J. Y. Kim, I. K. Park, K. S. Kim, G. Y. Jung, S. J. Park, J. W. Kim, and Y. C. Kim, "Enhanced emission efficiency of GaN/InGaN multiple quantum well light-emitting diode with an embedded photonic crystal”, Appl. Phys. Lett. 92, 251110 (2008).

[9] S. H. Han, D. Y. Lee, S. J. Lee, C. Y. Cho, M. K. Kwon, S. P. Lee, D. Y. Noh, D. J. Kim, Y. C. Kim, and S. J. Park, "Effect of electron blocking layer on efficiency droop in InGaN/GaN multiple quantum well light-emitting diodes", Appl. Phys. Lett. 94, 231123 (2009).

[10] C. C. Yang, C. F. Lin, C. M. Lin, C. C. Chang, K. T. Chen, J. F. Chien, and C. Y. Chang, "Improving light output power of InGaN-based light emitting diodes with pattern-nanoporous p-type GaN:Mg surfaces”, Appl. Phys. Lett. 93, 203103 (2008).

[11] K. Okamoto, I. Niki, A. Shvartser, Y. Narukawa, T. Mukai, and A. Scherer, "Surface-plasmon-enhanced light emitters based on InGaN quantum wells", Nature Mater. 3, 601 (2004).

[12] D. M. Yeh, C. Y. Chen, Y. C. Lu, C. F. Huang, and C. C. Yang, "Formation of various metal nanostructures with thermal annealing to control the effective coupling energy between a surface plasmon and an InGaN/GaN quantum well”, Nanotechnol. 18, 265402 (2007).

[13] T. S. Oh, H. Jeong, Y. S. Lee, J. D. Kim, T. H. Seo, H. Kim, A. H. Park, K. J. Lee, and E. K. Suh, "Coupling of InGaN/GaN multiquantum-wells photoluminescence to surface plasmons in platinum nanocluster", Appl. Phys. Lett. 95, $111112(2009)$. 
[14] Y. R. Wu, C. Chiu, C. Y. Chang, P. Yu, and H. C. Kuo, "Size-dependent strain relaxation and optical characteristics of InGaN/GaN nanorod LEDs", IEEE J. Quantum Electron. 15, 4 (2009).

[15] C. Hums, T. Finger, T. Hempel, J. Christen, and A. Dadgar, "Fabry-Perot effects in InGaN/GaN heterostructures on Si-substrate", J. Appl. Phys. 101, 033113 (2007).

[16] H. Kuwata, H. Tamaru, K. Esumi, and K. Miyano, "Resonant light scattering from metal nanoparticles: Practical analysis beyond Rayleigh approximation”, Appl. Phys. Lett. 83, 22 (2003).

[17] A. Neogi, C. W. Lee, H. O. Everitt, T. Kurota, A. Tackeuchi, and E. Yablonovitch, "Enhancement of spontaneous recombination rate in a quantum well by resonant surface plasmon coupling”, Phys. Rev. B 66, 153305 (2002).

[18] W. L. Barnes, A. Dereux, and T. W. Ebbesen, "Surface plasmon subwavelength optics", Nature 424, 824 (2003).

[19] O. A. Yeshchenko, I. M. Dmitruk, A. A. Alexeenko, M. Y. Losytskyy, A. V. Kotko, and A. O. Pinchuk, "Sizedependent surface-plasmon-enhanced photoluminescence from silver nanoparticles embedded in silica”, Phys. Rev. B 79, 235438 (2009).

[20] K. C. Lee, S. J. Lin, C. H. Lin, C. S. Tsai, and Y. J. Lu, "Size effect of Ag nanoparticles on surface plasmon resonance", Surf. Coat. Tech. 202, 5339-5342 (2008).

[21] C. H. Chiu, M. H. Lo, T. C. Lu, P. Yu, H. W. Huang, H. C. Kuo, and S, C. Wang, "Nano-processing techniques applied in GaN-based light-emitting devices with self-assembly Ni nano-masks", J. Lightwave Tech. 26, 11 (2008).

[22] E. M. Purcell, Phys. "Spontaneous emission probabilities at radio frequencies”, Rev. 69, 681 (1946). 


\section{Figures}

(a)

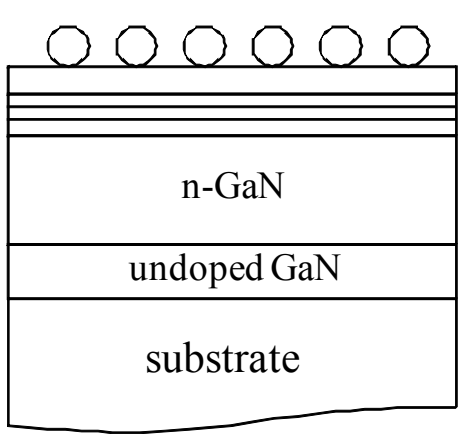

(b)

Ag cluster undoped GaN (10nm) MQWs

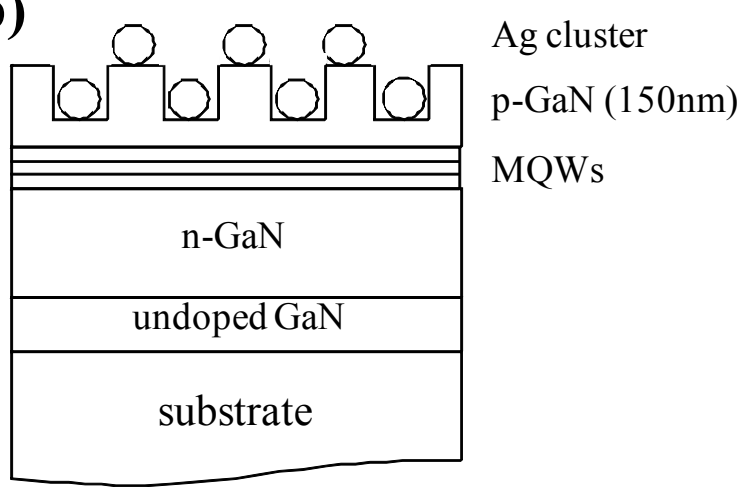

Fig. 1. Schematic sample structures for SP-QW coupling with $10 \mathrm{~nm}$ thick undoped $\mathrm{GaN}$ (a) and partially etched p-GaN (b). 

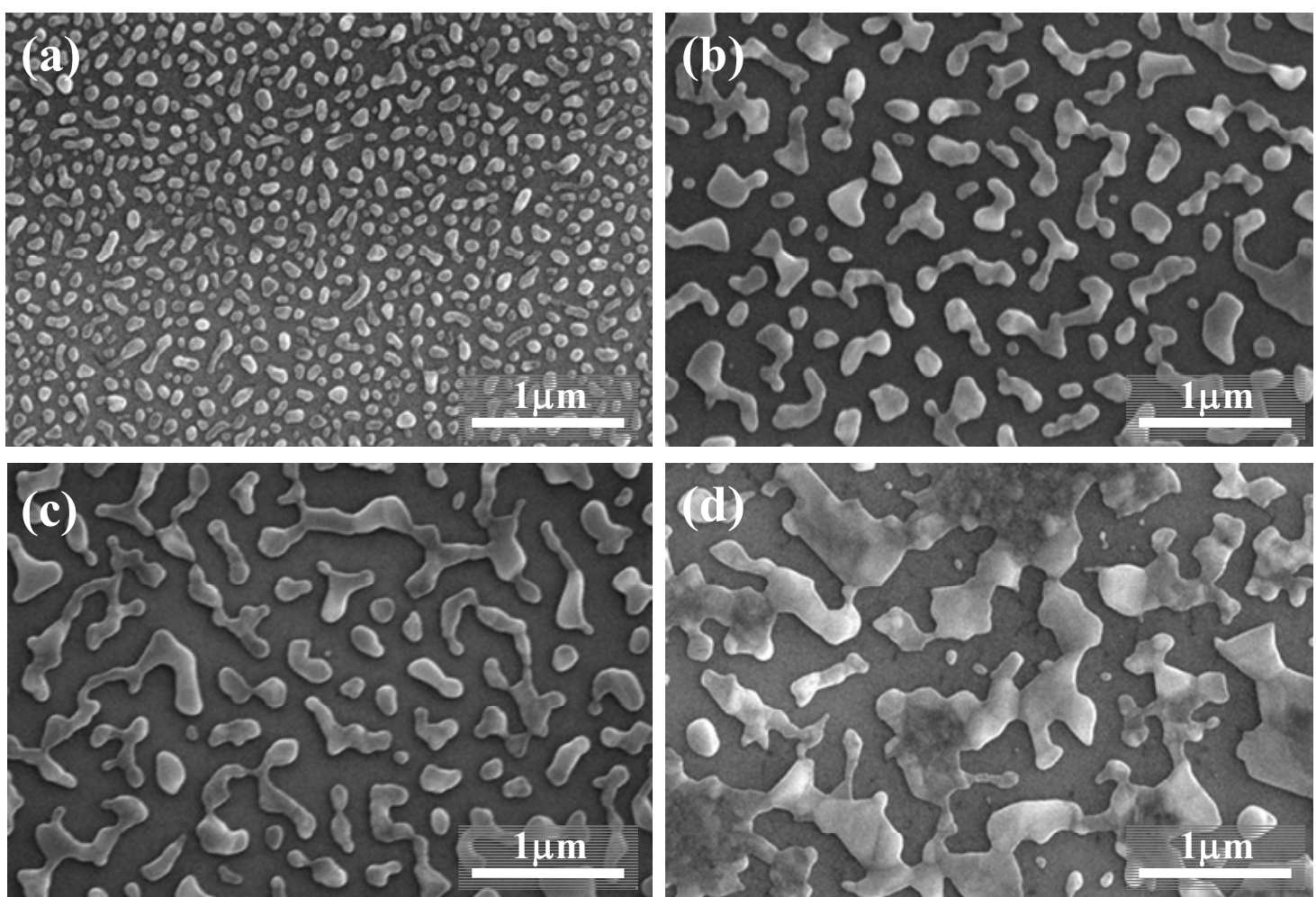

Fig. 2. SEM images of the Ag nanostructures formed on the $10 \mathrm{~nm}$ undoped GaN spacer after annealing the Ag films with thickness of $10 \mathrm{~nm}$ (a), $15 \mathrm{~nm}$ (b), $20 \mathrm{~nm}$ (c) and $25 \mathrm{~nm}(\mathrm{~d})$. 


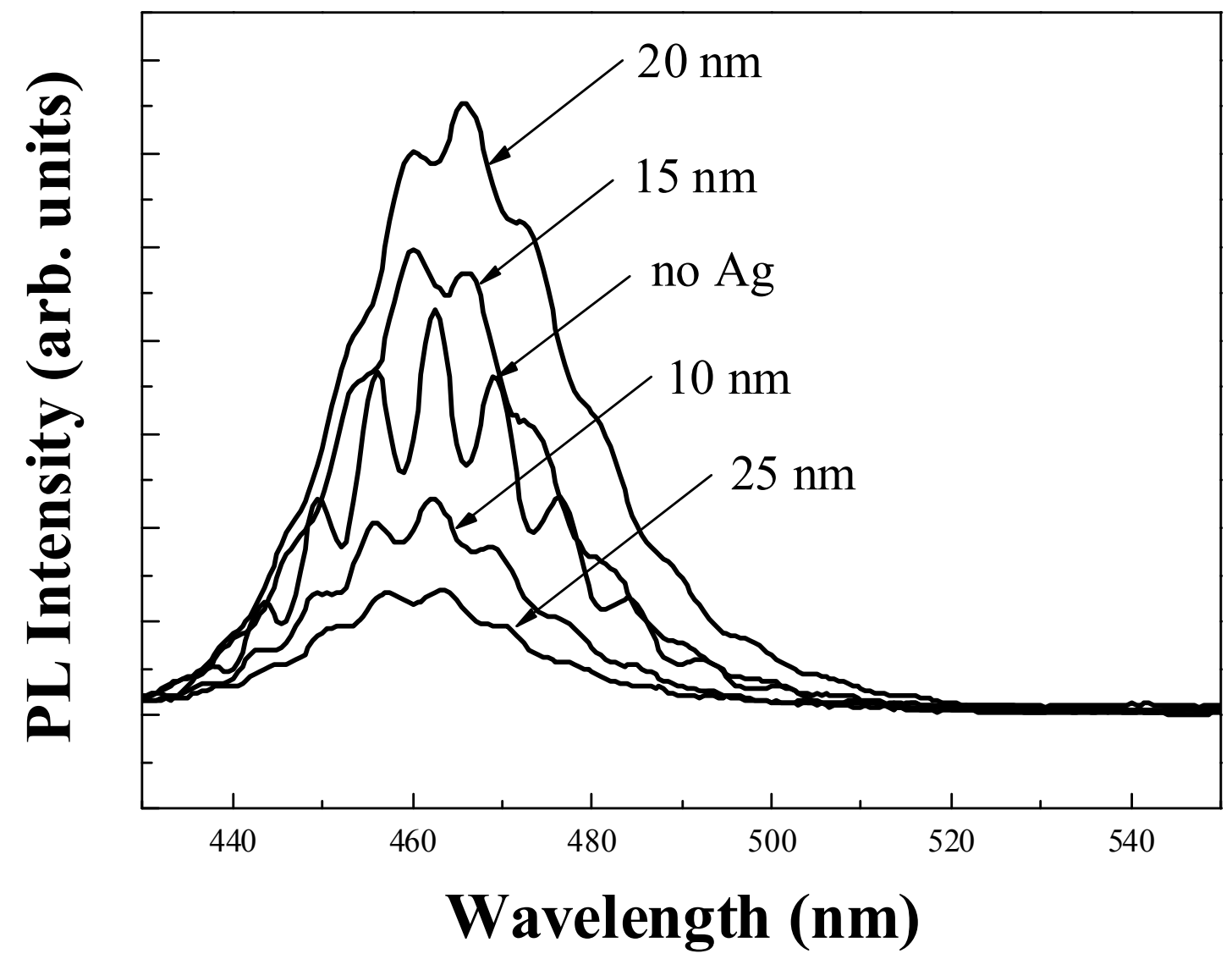

Fig. 3. Room temperature PL spectra from the samples with different Ag thickness. 


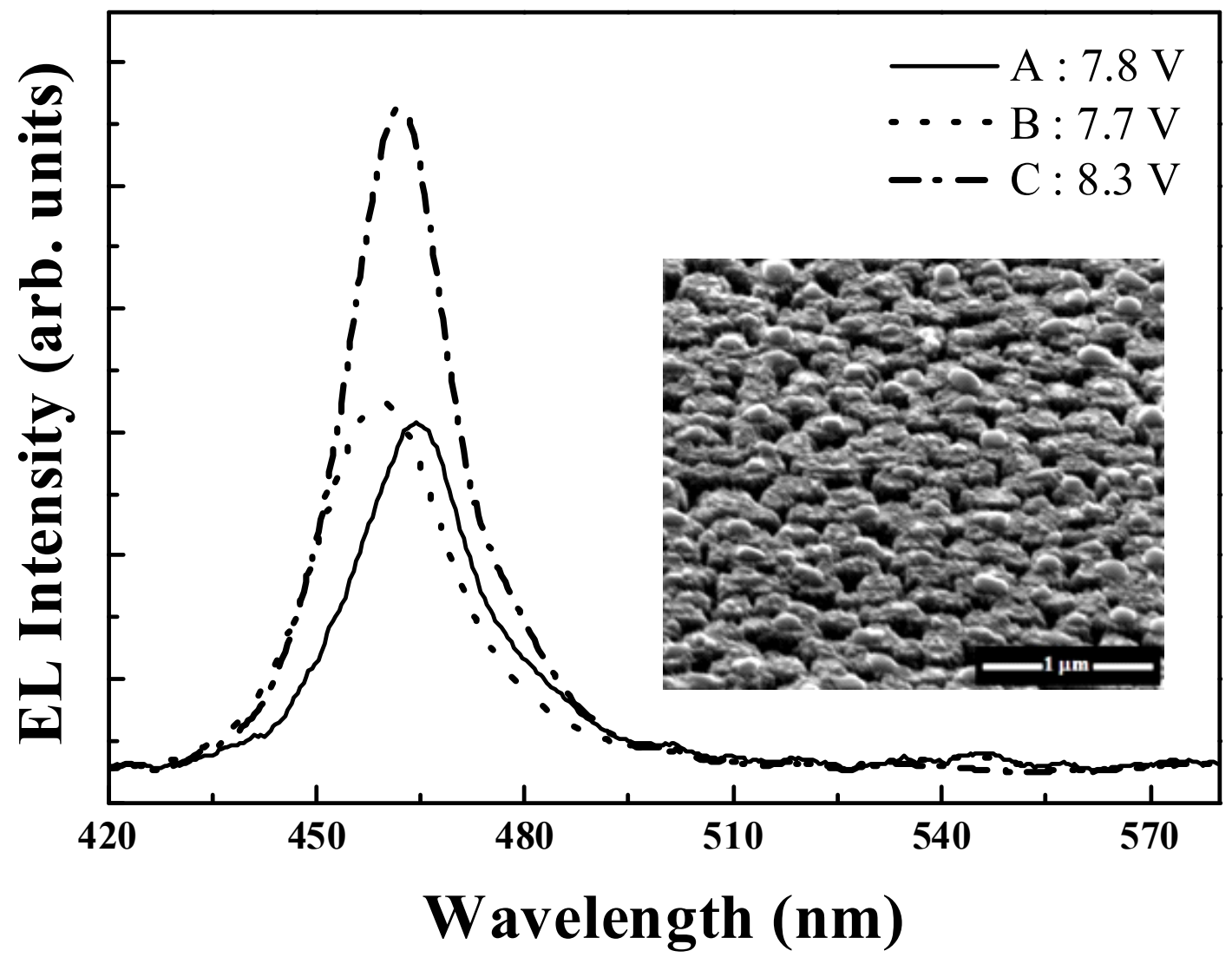

Fig. 4. EL spectra of the bare LED (A), ICP etched LED (B), and Ag coated LED after ICP etching (C). The inset shows the tilted surface morphology of the sample C by SEM. 


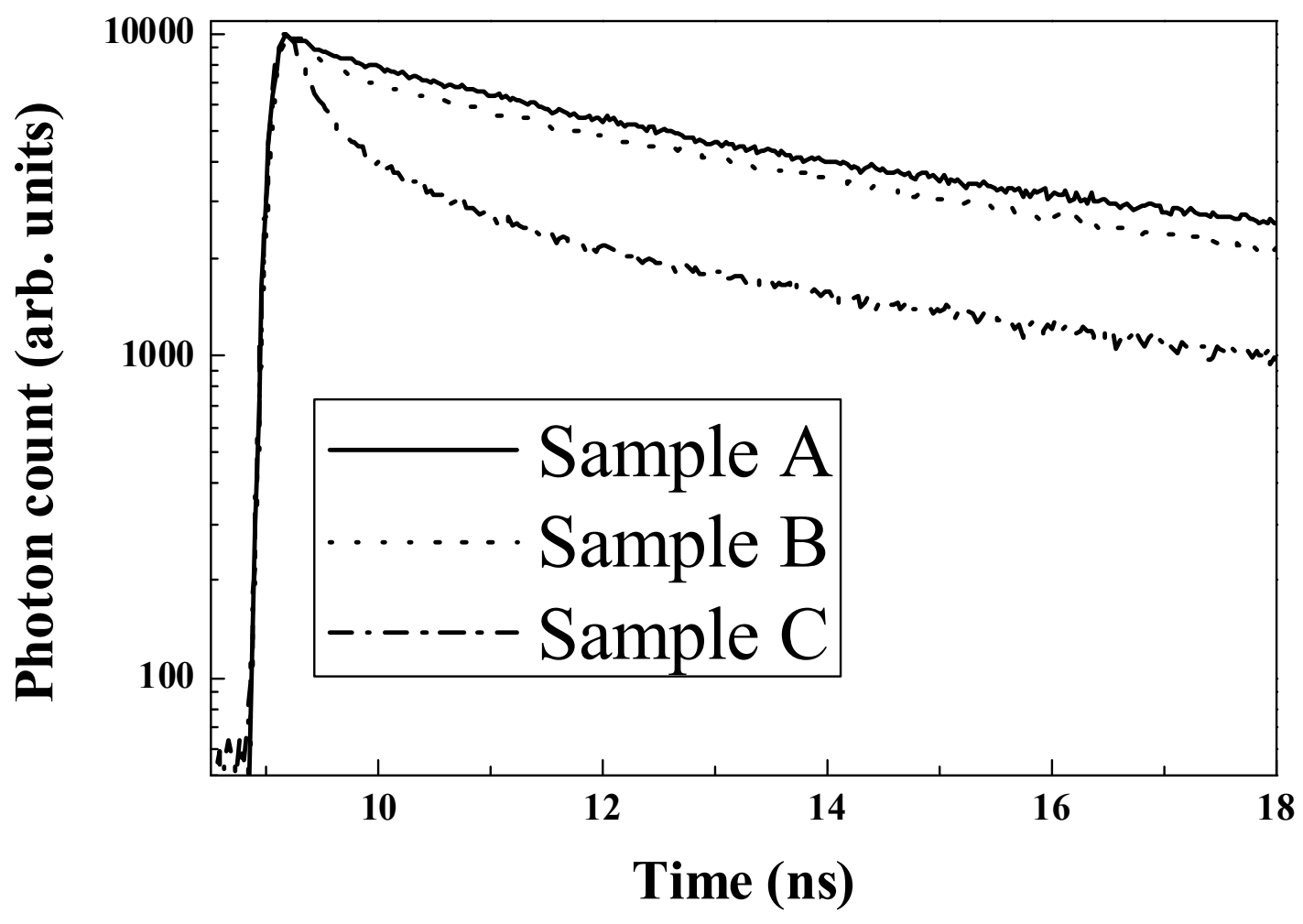

Fig. 5. Room temperature TRPL spectra of bare LEDs (A), ICP etched LEDs (B), and Ag coated LEDs after ICP etching (C). 